\title{
Quasi-Elastic Neutron Scattering Studies on Hydration Water in Phospholipid Membranes
}

\author{
Takeshi Yamada ${ }^{1}$ and Hideki Seto ${ }^{2 *}$ \\ ${ }^{1}$ Neutron Science and Technology Center, Comprehensive Research Organization for Science and Society, Tokai, Japan, \\ ${ }^{2}$ Institute of Materials Structure Science/J-PARC Center, High Energy Accelerator Research Organization, Tsukuba, Japan
}

\section{OPEN ACCESS}

Edited by:

Xi Zhang,

Shenzhen University, China

Reviewed by:

Giancarlo Franzese,

University of Barcelona, Spain

Luca Evangelisti,

University of Bologna, Italy

*Correspondence:

Hideki Seto

hideki.seto@kek.jp

Specialty section:

This article was submitted to Physical Chemistry and Chemical

Physics,

a section of the journal

Frontiers in Chemistry

Received: 18 October 2019

Accepted: 07 January 2020

Published: 24 January 2020

Citation:

Yamada $T$ and Seto $H$ (2020)

Quasi-Elastic Neutron Scattering

Studies on Hydration Water in

Phospholipid Membranes.

Front. Chem. 8:8.

doi: 10.3389/fchem.2020.00008
The dynamic behavior of hydration water in phospholipid membranes has been investigated to understand the relationship between water and biological molecules using various experimental techniques. Quasi-elastic neutron scattering (QENS) is an effective method for this purpose because the dynamic behaviors of both water and lipid molecules could be identified by using selective deuteration. In addition, the measurable ranges from the $10^{-12}$ to $10^{-9} \mathrm{~s}$ time scale and the $10^{-11}$ to $10^{-8} \mathrm{~m}$ length scale are suitable to investigate the slowing down of water molecules due to their interaction with lipid membranes. In this mini-review, QENS experiments on the dynamic behavior of hydration water molecules in neighboring phospholipid membranes are summarized.

Keywords: quasi elastic neutron scattering, biological membrane, phospholipid, dynamics, hydration

Water is essential for biological systems. For example, approximately $70 \%$ of the human body is water. This means that understanding the relationship between water and biological molecules is crucial to clarify the origins of biological functions. Many studies have been conducted on water and biological molecules such as DNA, proteins, and biological membranes (Bagchi, 2005; Berkowitz, 2019).

Many biological activities involve comprehensive transport processes through biological membranes. To understand these processes, gaining a full understanding of the structural and dynamic properties of biological membranes as well as surrounding water molecules is important. Because the composition and structure of a real biological membrane are too complex, simplified systems such as pure lipid membranes have been investigated as model systems. Because phospholipids are amphiphilic molecules, they naturally form into bilayers with hydrophobic tails inside and hydrophilic heads outside. These types of lipid membranes have been investigated using various types of experimental techniques such as nuclear magnetic resonance (NMR) (König et al., 1994; Hsieh and Wu, 1995; Nevzorov and Brown, 1997), X-ray diffraction (XRD) (Rand and Parsegain, 1989; Klose et al., 1992), neutron scattering (Pfeiffer et al., 1993; König et al., 1994; Rheinstädter et al., 2005, 2006; Seto et al., 2008; Nagao et al., 2017), dielectric spectroscopy (Antonietti et al., 1996; Klöesgen et al., 1996), differential scanning calorimetry (DSC) (Shalaev and Steponkus, 2003), dynamic light scattering (Hirn et al., 1999), and Fourier transform infrared spectroscopy (Wong and Mantsch, 1988). These experiments showed that the dynamics of membranes are hierarchical in spatial and temporal scales. It is also clear that all the dynamic behaviors strongly depend on the hydration of membranes.

To understand the relationship between phospholipid bilayers and water molecules, several experimental and computational studies have been conducted (Milhaud, 2004; Martelli et al., 2018; Calero and Franzese, 2019; Watanabe et al., 2019). Aoki and Kodama investigated the behavior of interlamellar water in phosphatidylethanolamine, phosphorylglycerol, and phosphocholine and 
showed the existence of both freezable and non-freezable water (Aoki and Kodama, 1998). Lazrak et al. revealed the water exchange between the inner part of the lipid membrane and the bulk region by NMR (Lazrak et al., 1987). The detailed structures of lipid membranes were shown, and the number of water molecules near the lipid headgroups were estimated through XRD (Nagle and Tristram-Nagle, 2000; Alsop et al., 2016). Molecular dynamics simulations showed that the hydration force and dynamic behavior of water molecules between lipid bilayers depend on the water structure (Marrink et al., 1993). Ultrafast polarization selective vibrational pump-probe spectroscopy of a stack of bilayers of phospholipid 1,2-dilauroyl-sn-glycero-3phosphocholine (DLPC) showed that the dynamic behavior of water molecules between lipid membranes was different from that of hydration water at the phosphate and choline groups (Zhao et al., 2008). In another study, femtosecond mid-IR pumpprobe spectroscopy verified that the structure and dynamics of water between 1,2-dimyristyl-sn-glycero-3-phosphocholine (DMPC) membranes varied with the phase transition of the DMPC bilayer from the gel phase to the liquid crystalline phase (Kundu et al., 2016). Another study investigated the dynamic behavior of water near the DMPC membranes by $\mathrm{THz}$ spectroscopy, and it was shown that 28 water molecules were hydrated per DMPC molecule, which was greater than the previously reported value (Hishida and Tanaka, 2011). Other $\mathrm{THz}$ spectroscopy experiments showed that the ratio of hydration water molecules against the total number of water molecules increased during the transition from the gel to the liquid crystalline phase (Choi et al., 2012).

Of the various types of experimental techniques, quasi-elastic neutron scattering (QENS) is a powerful method to investigate the dynamic behavior of water molecules from the $10^{-12}$ to $10^{-9}$ $\mathrm{s}$ time scale and from the $10^{-11}$ to $10^{-8} \mathrm{~m}$ length scale through the dynamic structure factor $S(Q, \omega)$. Because hydrogen has a huge incoherent scattering cross section throughout the atoms, neutron scattering from materials containing rich hydrogen is dominated by incoherent scattering. Although this incoherent scattering does not include the static structure, it provides information on the self-correlated dynamics of hydrogen such as the self-diffusion coefficient and rotational relaxation time (Teixeira et al., 1985; Amann-Winkel et al., 2016). The coherent and incoherent neutron scattering cross sections of deuterium are different from those of hydrogen, where the labeling of hydrogen can be performed by selective deuteration. Thus, the appropriate deuteration is useful to investigate the structural and dynamic properties of mixed compounds such as water included in materials.

Physical properties of water have attracted considerable attention for years because water shows specific features not found in other simple liquids, including a maximum density at $4^{\circ} \mathrm{C}$ (Zheleznyi, 1969) and divergence of heat capacity in the super-cooled region (Angell et al., 1982; Debenedetti, 2003). To elucidate the origin of the aforementioned specific features, Poole et al. showed the possible existence of a second critical point below the homogeneous nucleation temperature by molecular dynamics simulation (Poole et al., 1992). Both dynamic and structural behaviors of confined water in mesoporous materials such as mesoporous silicate have been investigated by neutron scattering because the crystallization is considered to be suppressed in the nanometer-scale space. Liu et al. reported the pressure and temperature dependence of the translational relaxation time of confined water in MCM-41 (Liu et al., 2005). Here, the translational relaxation time showed a strongfragile transition. Yoshida et al. also reported the strong-fragile transition of confined water in MCM-41 (Yoshida et al., 2008). This transition is discussed with a hypothesis for the second critical point of water as previously described (Poole et al., 1992). The experimental results have been summarized in reviews (Mishima and Stanley, 1998; Debenedetti, 2003; Cerveny et al., 2016).

In addition, hydration water at the interface has been recognized to play a major role in functional and biological materials. Perrin et al. reported the dynamics of hydrated water in the Nafion ${ }^{\circledR}$ membrane, which is one of the most popular proton conductors, and showed the existence of two types of water characterized by local and long-range diffusion (Perrin et al., 2007). Copper rubeanate, which is a porous coordinate metal complex, exhibits high proton conductivity under high relative humidity conditions (Kitagawa et al., 2003). Yamada et al. reported that the adsorbed water in the copper rubeanate pore could be categorized into "free water," the diffusion coefficient of which was similar to that of bulk water, and "condensed water," the diffusion coefficient of which is 10 times slower than that of free water (Yamada et al., 2011). The free water was condensed on the pore surface with the first-order transition at $260 \mathrm{~K}$, and the transition was a type of liquid-liquid transition. The dynamics of the water was slowed down due to the steric hindrance in the case of hydroxyethyl copper rubeanate (Yamada et al., 2013). Noferini et al. investigated the dynamics of hydration water in polyhydroxyethyl methacrylate gels (Noferini et al., 2019). The hydrated water was separated into immobile water associated with the polymer matrix and mobile water confined in a gel matrix. Russina et al. reported the water dynamics in a hydrophobic pore of aluminophosphate $\mathrm{AlPO}_{4}-5$, which provided a one-dimensional hydrophobic channel (Russina et al., 2019). The water had two diffusive motions: fast diffusion, which corresponded to the position exchange between neighboring water molecules, and slow diffusion, which corresponded to the long-range diffusion along the pore channel. These studies clearly indicated that QENS has advantages in exploring the hierarchy of the dynamics of hydration water.

As described above, the incoherent and coherent scattering cross sections are different between hydrogen and deuterium. Thus, selective deuteration enables identifying the structure and dynamics of water and phospholipid molecules separately. The first QENS results on the dynamic behavior of water molecules between lipid bilayers were published in 1994. König et al. examined oriented perdeuterated 1,2-dipalmitoyl-sn-glycero-3phosphocholine bilayers (DPPC) at two hydration levels $\left(n_{w}=\right.$ $4-5$ and 11) (König et al., 1994). Two membrane orientations (i.e., where the scattering vector was normal and parallel to the membrane) were selected to observe the anisotropy of the water dynamics in the membrane. These experiments were performed using the IRIS spectrometer at ISIS, UK, at an energy resolution of $15 \mu \mathrm{eV}$. The researchers showed that water molecules exhibited a slow rotational motion and no translational 
diffusion in the low hydration state. Jump diffusion motion similar to that of bulk water was identified in the high hydration state. The experimental results revealed homogeneous dynamic behavior, and anisotropy was not observed.

The results of the QENS experiments with selective deuteration were shown by Swenson et al. (2008). They prepared three samples: fully protonated DMPC with heavy water $\left(\mathrm{DMPC} / \mathrm{D}_{2} \mathrm{O}\right)$, acyl-chain deuterated DMPC with heavy water (d54-DMPC/ $\left.\mathrm{D}_{2} \mathrm{O}\right)$, and acyl-chain deuterated DMPC with light water $\left(\mathrm{d} 54-\mathrm{DMPC} / \mathrm{H}_{2} \mathrm{O}\right)$. QENS experiments were also performed with the IRIS spectrometer at a $17-\mu \mathrm{eV}$ energy resolution. The solutions were deposited on $\mathrm{Si}(111)$ wafers to determine the orientation difference. The dynamic behavior of water molecules was obtained by subtracting the QENS data of $\mathrm{d} 54-\mathrm{DMPC} / \mathrm{D}_{2} \mathrm{O}$ from that of $\mathrm{d} 54-\mathrm{DMPC} / \mathrm{H}_{2} \mathrm{O}$. The $S(Q, \omega)$ data were converted into the intermediate scattering function $I(Q, t)$, and described through the Kohlrausch-Williams-Watts (KWW) stretched exponential relaxation function. From the $Q$ dependence of the relaxation time, the researchers concluded that the relaxation process was that of jump diffusion, and the diffusion constant was lower than that of bulk water by only a factor of two. This was attributed to the limited energy resolution of the experiment as well as the failure to approach the subnanosecond time scale. No significant directional dependence was observed.

Rheinstädter et al. published the results of high-resolution QENS experiments with selective deuteration in 2008 (Rheinstädter et al., 2008). They deposited organic solution of acyl-chain deuterated DMPC (d54-DMPC) or fully protonated DMPC on $\mathrm{Si}$ wafers and hydrated with $\mathrm{D}_{2} \mathrm{O}$ or $\mathrm{H}_{2} \mathrm{O}$ vapor after drying. They measured four samples, d54-DMPC/ $\mathrm{D}_{2} \mathrm{O}$, d54-DMPC/ $\mathrm{H}_{2} \mathrm{O}, \mathrm{DMPC} / \mathrm{D}_{2} \mathrm{O}$, and $\mathrm{DMPC} / \mathrm{H}_{2} \mathrm{O}$, using the cold neutron backscattering spectrometer IN16 at the Institute Laue-Langevin, France, with an energy resolution of 0.9 $\mu \mathrm{eV}$. The QENS data were analyzed with the aid of an allatom MD simulation of DMPC, which showed a cooperative structural relaxation process in fluid membranes over several lipid distances.

The subsequent results of this group on the dynamic behavior of hydration water molecules were published in 2015 (Toppozini et al., 2015). The researchers prepared oriented d54-DMPC layers on $\mathrm{Si}$ wafers hydrated with $\mathrm{H}_{2} \mathrm{O}$ vapor and performed QENS experiments with the LET spectrometer at ISIS. They also analyzed the QENS data using the KWW function. They concluded that the dynamics of hydration water molecules is anisotropic and exhibit a sub-diffusive behavior in nanometerlength scales.

The most recent results were published in 2017 by Yamada et al. (2017). They prepared two samples, perdeuterated DMPC (d67-DMPC) with $\mathrm{H}_{2} \mathrm{O}$ and protonated DMPC with $\mathrm{D}_{2} \mathrm{O}$ to compare the dynamic behaviors of water and lipid molecules. The DMPC powder was mixed with the appropriate amount of water to obtain a ratio of 37 water molecules for each DMPC molecule, which corresponded to nearly the maximum amount water incorporated between lipid bilayers (Hishida and Tanaka, 2011). In contrast to the experiments previously described, the mixtures were wrapped with aluminum foil and placed in an aluminum cylinder cell. The QENS experiments were performed using the DNA spectrometer in MLF, J-PARC, Japan, at an energy resolution of $3.6 \mu \mathrm{eV}$. Data analysis showed that the hydration water could be categorized into three types: (1) free water with dynamic behavior is slightly different from that of bulk water; (2) loosely bound water with dynamic behavior is one-order of magnitude slower than that of free water; and (3) tightly bound water with dynamic behavior is comparable to that of DMPC molecules. The slow dynamics of loosely and tightly bound water were also reported based on molecular dynamics simulation, where the bound water forming strong hydrogen bonds to DMPC were observed to have a 20-times smaller translational diffusion coefficient than that of bulk water (Calero and Franzese, 2019). The number of the free water was 23 , and the activation energy of the free water was smaller than that of bulk water. It could be related to the intermediate range order of the hydrated water reported by Martelli et al. (2018). The sum of the tightly and loosely bound water was 14 , and the fraction depended on temperature. These results were quantitatively consistent with those measured by DSC (Aoki and Kodama, 1998) and by terahertz spectroscopy (Hishida and Tanaka, 2011).

QENS experiments on water and lipid membranes have the potential to relate the dynamic and structural properties of water and the functions of biological molecules. In addition, it has been known that the existence of a significant amount of loosely bound water, also referred to as "intermediate water" or "freezable water" in the literature, is considered a key factor in characterizing bio-compatible polymers (Tsuruta, 2010). The aforementioned results suggest that QENS could be a major tool used to clarify the origin of bio-related functions in biology and in material science.

\section{AUTHOR CONTRIBUTIONS}

All authors listed have made a substantial, direct and intellectual contribution to the work, and approved it for publication.

\section{FUNDING}

This work was supported by JSPS KAKENHI, JP18K11932, and a Grant-in-Aid for Scientific Research on Innovative Areas No. JP19H05717 (Aquatic Functional Materials).

\section{REFERENCES}

Alsop, R. J., Schober, R. M., and Rheinstädter, M. C. (2016). Swelling of phospholipid membranes by divalent metal ions depends on the location of the ions in the bilayers. Soft Matt. 12, 6737-6748. doi: 10.1039/C6SM0 $0695 \mathrm{G}$

Amann-Winkel, K., Bellissent-Funel, M.-C., Bove, L. E., Loerting, T., Nilsson, A., Paciaroni, A., et al. (2016). X-ray and neutron scattering of water. Chem. Rev. 116, 7570-7589. doi: 10.1021/acs.chemrev.5b00663

Angell, C. A., Sichina, W. J., and Oguni, M. (1982). Heat capacity of water at extremes of supercooling and superheating. J. Phys. Chem. 86, 998-1002. doi: $10.1021 / \mathrm{j} 100395 \mathrm{a} 032$ 
Antonietti, M., Neese, M., Blum, G., and Kremer, F. (1996). Dielectric and mechanic relaxation in polyelectrolyte-supported bilayer stacks: a model for the dynamics of membranes? Langmuir 12:4436. doi: 10.1021/la960221b

Aoki, H., and Kodama, M. (1998). Calorimetric investigation of the behavior of interlamellar water in phospholipid-water systems. Thermochim. Acta 308:77. doi: 10.1016/S0040-6031(97)00334-1

Bagchi, B. (2005). Water dynamics in the hydration layer around proteins and micelles. Chem. Rev. 105, 3197-3219. doi: 10.1021/cr020661+

Berkowitz, M. L. (2019). Biomembrane Simulations. Boca Raton, FL: CRC Press.

Calero, C., and Franzese, G. (2019). Membranes with different hydration levels: the interface between bound and unbound hydration water. J. Mol. Liquids 273, 488-496. doi: 10.1016/j.molliq.2018.10.074

Cerveny, S., Mallamace, F., Swenson, J., Vogel, M., and Xu, L. (2016). Confined water as model of supercooled water. Chem. Rev. 116, 7608-7625. doi: 10.1021/acs.chemrev.5b00609

Choi, D.-H., Son, H., Jung, S., Park, J., Park, W.-Y., Kwon, O. S., et al. (2012). Dielectric relaxation change of water upon phase transition of a lipid bilayer probed by terahertz time domain spectroscopy. J. Chem. Phys. 137:175101. doi: $10.1063 / 1.4764304$

Debenedetti, P. G. (2003). Supercooled and glassy water. J. Phys. 15, R1669-R1726. doi: 10.1088/0953-8984/15/45/R01

Hirn, R., Bayerl, T., Rädler, J., and Sackmann, E. (1999). Collective membrane motions of high and low amplitude, studied by dynamic light scattering and micro-interferometry. Faraday Discuss. 111:17. doi: 10.1039/a807883a

Hishida, M., and Tanaka, K. (2011). Long-range hydration effect of lipid membrane studied by terahertz time-domain spectroscopy. Phys. Rev. Lett. 106:158102. doi: 10.1103/PhysRevLett.106.158102

Hsieh, C. H., and Wu, W. G. (1995). Solvent effect on phosphatidylcholine headgroup dynamics as revealed by the energetics and dynamics of two gelstate bilayer headgroup structures at subzero temperatures. Biophys. J. 69:4. doi: 10.1016/S0006-3495(95)79885-X

Kitagawa, H., Nagao, Y., Fujishima, M., Ikeda, R., and Kanda, S. (2003). Highly proton-conductive copper coordination polymer, h2dtoacu (h2dtoa=dithiooxamide anion). Inorg. Chem. Commun. 6, 346-348. doi: 10.1016/S1387-7003(02)00749-9

Klöesgen, B., Reichle, C., Kohlsmann, S., and Kramer, K. D. (1996). Dielectric spectroscopy as a sensor of membrane headgroup mobility and hydration. Biophys. J. 71:3251. doi: 10.1016/S0006-3495(96)79518-8

Klose, G., König, B., and Paltauf, F. (1992). Sorption isotherms and swelling of popc in h2o and 2h2o. Chem. Phys. Lipids 61:265. doi: 10.1016/0009-3084(92)90106-Y

König, S., Sackmann, E., Richter, D., Zorn, R., Carlile, C., and Bayerl, T. M. (1994). Molecular dynamics of water in oriented dppc multilayers studied by quasielastic neutron scattering and deuterium-nuclear magnetic resonance relaxation. J. Chem. Phys. 100, 3307-3316. doi: 10.1063/1.466422

Kundu, A., Blasiak, B., Lim, J.-H., Kwak, K., and Cho, M. (2016). Water hydrogenbonding network structure and dynamics at phospholipid multibilayer surface: femtosecond mid-ir pump-probe spectroscopy. J. Phys. Chem. Lett. 7, 741-745. doi: 10.1021/acs.jpclett.6b00022

Lazrak, T., Milon, A., Wolff, G., Albrecht, A.-M., and Nakatani, Y. (1987). Comparison of the effects of inserted c40- and c50-terminally dihydroxylated carotenoids on the mechanical properties of various phospholipid vesicles. Biochim. Biophys. Acta 903, 132-141. doi: 10.1016/0005-2736(87)90163-5

Liu, L., Chen, S.-H., Faraone, A., Yen, C.-W., and Mou, C.-Y. (2005). Pressure dependence of fragile-to-strong transition and a possible second critical point in supercooled confined water. Phys. Rev. Lett. 95:117802. doi: 10.1103/PhysRevLett.95.117802

Marrink, S. J., Berkowitz, M., and C., B. H. J. (1993). Molecular dynamics simulation of a membrane/water interface: the ordering of water and its relation to the hydration force. Langmuir 9, 3122-3131. doi: 10.1021/la00035a062

Martelli, F., Ko, H.-Y., Borallo, C. C., and Franzese, G. (2018). Structural properties of water confined by phospholipid membranes. Front. Phys. 13:136801. doi: $10.1007 /$ s11467-017-0704-8

Milhaud, J. (2004). New insites into water-phospholipid model membrane interactions. Biochim. Biophys. Acta 1663, 19-51. doi: 10.1016/j.bbamem.2004.02.003

Mishima, O., and Stanley, H. E. (1998). The relationship between liquid, supercooled and glassy water. Nature 396, 329-335. doi: 10.1038/24540
Nagao, M., Kelly, E. G., Ashkar, R., Bradbury, R., and Butler, P. D. (2017). Probing elastic and viscous properties of phospholipid bilayers using neutron spin echo spectroscopy. J. Phys. Chem. Lett. 8, 4679-4684. doi: 10.1021/acs.jpclett.7b01830

Nagle, J. F., and Tristram-Nagle, S. (2000). Structure of lipid bilayers. Biochim. Biophys. Acta 1469, 159-195. doi: 10.1016/S0304-4157(00)00016-2

Nevzorov, A., and Brown, M. (1997). Dynamics of lipid bilayers from comparative analysis of $2 \mathrm{~h}$ and $13 \mathrm{c}$ nuclear magnetic resonance relaxation data as a function of frequency and temperature. J. Chem. Phys. 107:10288. doi: 10.1063/1.474169

Noferini, D., Faraone, A., Rossi, M., Mamontov, E., Fratini, E., and Baglioni, P. (2019). Disentangling polymer network and hydration water dynamics in polyhydroxyethyl methacrylate physical and chemical hydrogels. J. Phys. Chem. C 123, 19183-19194. doi: 10.1021/acs.jpcc.9b04212

Perrin, J.-C., Lyonnard, S., and Volino, F. (2007). Quasielastic neutron scattering study of water dynamics in hydrated nafion membranes. J. Phys. Chem. C 111, 3393-3404. doi: 10.1021/jp065039q

Pfeiffer, W., König, S., Legrand, J., Bayerl, T., Richter, D., and Sackermann, E. (1993). Neutron spin echo study of membrane undulations in lipid multibilayers. Europhys. Lett. 23:457. doi: 10.1209/0295-5075/23/6/013

Poole, P. H., Sciortino, F., Essmann, U., and Stanley, H. E. (1992). Phase behaviour of metastable water. Nature 360:324. doi: 10.1038/360324a0

Rand, R. P., and Parsegain, V. A. (1989). Hydration forces between phospholipid bilayers. Biochim. Biophys. Acta 988:351. doi: 10.1016/0304-4157(89)90010-5

Rheinstädter, M. C., Das, J., Flenner, E. J., Brüning, Seydel, T., and Kosztin, I. (2008). Motional coherence in fluid phospholipid membranes. Phys. Rev. Lett. 101:248106. doi: 10.1103/PhysRevLett.101.248106

Rheinstädter, M. C., Häußler, H., and Salditt, T. (2006). Dispersion relation of lipid membrane shape fluctuations by neutron spin-echo spectrometry. Phys. Rev. Lett. 101:048103. doi: 10.1103/PhysRevLett.97.048103

Rheinstädter, M. C., Seydel, T., Demmel, F., and Salditt, T. (2005). Molecular motions in lipid bilayers studied by the neutron backscattering technique. Phys. Rev. E 71:061908. doi: 10.1103/PhysRevE.71.061908

Russina, M., Günther, G., Grzimek, V., Schlegel, M. C., Veziri, C. M., Karanikolos, G. N., et al. (2019). Nanoscale dynamics and transport in highly ordered low-dimensional water. J. Phys. Chem. Lett. 10, 6339-6344. doi: 10.1021/acs.jpclett.9b02303

Seto, H., Yamada, N. L., Nagao, M., Hishida, M., and Takeda, T. (2008). Bending modulus of lipid bilayers in liquid-crystalline phase including anomalous swelling regime estimated by nse experiments. Euro. Phys. J. E 26, 217-223. doi: 10.1140/epje/i2007-10315-0

Shalaev, E. Y., and Steponkus, P. L. (2003). Glass transition of a synthetic phospholipid in the lamellar phase. J. Phys. Chem. B 107:8734. doi: 10.1021/jp0303265

Swenson, J., Kargl, F., P., B., and C., S. (2008). Solvent and lipid dynamics of hydrated lipid bilayers by incoherent quasielastic neutron scattering. J. Chem. Phys. 129:045101. doi: 10.1063/1.2955753

Teixeira, J., Bellissent-Funel, M.-C., Chen, S. H., and Dianoux, A. J. (1985). Experimental determination of the nature of diffusive motions of water molecules at low temperatures. Phys. Rev. A 31, 1913-1917. doi: 10.1103/PhysRevA.31.1913

Toppozini, L., Roosen-Runge, F., Bewley, R. I., Dalgliesh, M., R., Perring, T., Seydel, T., et al. (2015). Anomalous and anisotropic nanoscale diffusion of hydration water molecules in fluid lipid membranes. Soft Matt. 11:8534. doi: 10.1039/C5SM01713K

Tsuruta, T. (2010). On the role of water molecules in the interface between biological systems and polymers. J. Biomat. Sci. 21, 1831-1848. doi: 10.1163/092050610X488269

Watanabe, N., Suga, K., and Umakoshi, H. (2019). Functional hydration behavior: interrelation betweenhydration and molecular properties at lipid membrane interface. Hindawi J. Chem. 2019:4867327. doi: 10.1155/2019/4867327

Wong, P. T. T., and Mantsch, H. H. (1988). High-pressure infrared spectroscopic evidence of water binding sites in 1,2-diacyl phospholipids. Chem. Phys. Lipids 46:213. doi: 10.1016/0009-3084(88)90024-2

Yamada, T., Takahashi, N., Tominaga, T., Takata, S., and Seto, H. (2017). Dynamical behavior of hydration water molecules between phospholipid membranes. J. Phys. Chem. B 121, 8322-8329. doi: 10.1021/acs.jpcb.7b01276

Yamada, T., Yamada, T., Tyagi, M., Nagao, M., Kitagawa, H., and Yamamuro, O. (2013). Phase transition and dynamics of water confined in hydroxyethyl 
copper rubeanate hydrate. J. Phys. Soc. Jpn. 82(Suppl. A):SA010. doi: $10.7566 /$ JPSJS.82SA.SA010

Yamada, T., Yonamine, R., Yamada, T., Kitagawa, H., Tyagi, M., Nagao, M., et al. (2011). Quasi-elastic neutron scattering studies on dynamics of water confined in nanoporous copper rubeanate hydrates. J. Phys. Chem. B 115, 13563-13569. doi: $10.1021 /$ jp2029467

Yoshida, K., Yamaguchi, T., Kittaka, S., Bellissent-Funel, M.-C., and Fouquet, P. (2008). Thermodynamic, structural, and dynamic properties of supercooled water confined in mesoporous $\mathrm{mcm}-41$ studied with calorimetric, neutron diffraction, and neutron spin echo measurements. J. Chem. Phys. 129:054702. doi: $10.1063 / 1.2961029$

Zhao, W., Moilanen, D. E., Fenn, E. E., and Fayer, M. D. (2008). Water at the surfaces of aligned phospholipid multibilayer model membranes probed with ultrafast vibrational spectroscopy.
J. Am. Chem. Soc. 130, 13927-13937. doi: 10.1021/ja8 $03252 \mathrm{y}$

Zheleznyi, B. V. (1969). Density of supercooled water. Russ. J. Phys. Chem. 43:1311.

Conflict of Interest: The authors declare that the research was conducted in the absence of any commercial or financial relationships that could be construed as a potential conflict of interest.

Copyright (ㄷ 2020 Yamada and Seto. This is an open-access article distributed under the terms of the Creative Commons Attribution License (CC BY). The use, distribution or reproduction in other forums is permitted, provided the original author(s) and the copyright owner(s) are credited and that the original publication in this journal is cited, in accordance with accepted academic practice. No use, distribution or reproduction is permitted which does not comply with these terms. 